\title{
Neural Networks-Based Identification and Control of a Large Flexible Antenna
}

\author{
Minoru Sasaki, ${ }^{1}$ Takuya Murase, ${ }^{1}$ Yoshihiro Inoue, ${ }^{2}$ and Nobuharu Ukita ${ }^{3}$ \\ ${ }^{1}$ Department of Human and Information Systems Engineering, Gifu University, Gifu 501-1193, Japan \\ ${ }^{2}$ Department of Mechanical Systems Engineering, Gifu University, Gifu 501-1193, Japan \\ ${ }^{3}$ National Astronomical Observatory of Japan, Tokyo 181-0015, Japan \\ Correspondence should be addressed to Takuya Murase, h3132040@guedu.cc.gifu-u.ac.jp \\ Received 21 June 2011; Accepted 8 August 2011 \\ Academic Editor: S. Marchesiello
}

Copyright (c) 2011 Minoru Sasaki et al. This is an open access article distributed under the Creative Commons Attribution License, which permits unrestricted use, distribution, and reproduction in any medium, provided the original work is properly cited.

This paper presents identification and control of a 10-m antenna via accelerometers and angle encoder data. Artificial neural networks can be used effectively for the identification and control of nonlinear dynamical system such as a large flexible antenna with a friction drive system. Some identification results are shown and compared with the results of conventional prediction error method. And we use a neural network inverse model to control the large flexible antenna. In the neural network inverse model, a neural network is trained, using supervised learning, to develop an inverse model of the antenna. The network input is the process output, and the network output is the corresponding process input. The control results show the validation of the ANN approach for identification and control of the 10-m flexible antenna.

\section{Introduction}

This paper presents identification and control of a $10-\mathrm{m}$ antenna dynamics using accelerometers and angle encoders data.

ALMA — the Atacama large millimeter array-will be a single instrument composed of 64 high-precision antennas located on the Chajnantor plain of the Chilean Andes in the District of San Pedro de Atacama, 16,500 feet (5,000 meters) above sea level (shown in Figure 1). ALMA's primary function will be to observe and image with unprecedented clarity the enigmatic cold regions of the universe, which are optically dark yet shine brightly in the millimeter portion of the electromagnetic spectrum.

The ALMA is an international collaboration between Europe and the North America to build a synthesis radio telescope that will operate at millimeter and submillimeter wavelengths. Japan also becomes a partner, making this a truly global collaboration.

Its main targets include planetary system formation and galaxy formation/evolution. The technical challenges to key instruments for such arrays are now performed, that is, developments of high precision antenna, low-noise submillimeter mixers, high-power submillimeter LO sources, and very high-speed samplers and wideband spectrocorrelators. The specifications imposed for recent submillimeter antennas of a 10/12-m size in the open air are demanding and challenging. For example, 12-m antennas for Atacama large millimeter/submillimeter Array (ALMA) have a surface accuracy of better than $25 \mu \mathrm{m}$ and pointing/tracking accuracy of better than 0.6 " under a wind velocity of $9 \mathrm{~m} \mathrm{~s}-1$. They must also be able to slew to new position 1.5 degrees away and settle to within 3 arcsec in less than $1.5 \mathrm{sec}$ to cope with phase errors caused by fluctuations in the atmosphere.

Loads on antenna structure due to wind cause elastic deformations, which deteriorate antenna's pointing and surfaces accuracies. The structural behavior of the telescope is typically measured at the encoders of azimuth and elevation axes, while the critical performance is the actual pointing on the sky. We need to make direct measurements of vibration motion of the main-dish and subreflector with a resolution of typically $3-5 \mu \mathrm{m}$. Seismic accelerometers serve this purpose for a frequency range from 0.1 to $100 \mathrm{~Hz}$. A laser metrology system can also serve for a frequency range $<1 \mathrm{~Hz}$. 


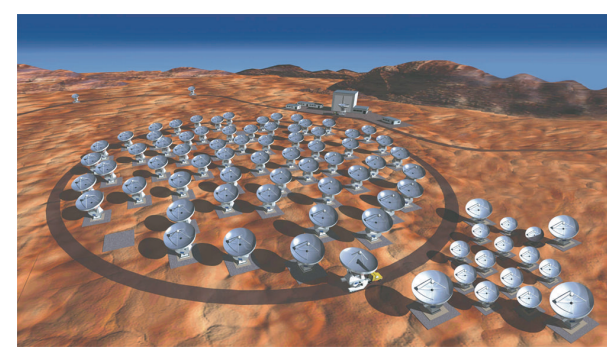

Figure 1: Rending of ALMA.

The low-frequency component $(<1 \mathrm{~Hz})$ is presumably due to wind load and the high frequency $(>1 \mathrm{~Hz})$ due to modal oscillation induced by a servo controller. It is very hard to identify and control of the antenna system [1-9].

With all advanced control schemes, mathematical knowledge of the dynamics of the process of interest is necessary. System identification refers to the process of developing a mathematical process model from experimental data. System identification in control engineering is a key element for understanding and controlling unknown dynamical systems. Traditional system identification techniques such as least square estimation, quasilinearization and stochastic modeling have been successfully used in nonlinear dynamical systems. In traditional system, model structure must be defined a priori to estimate all required system parameters. In case of antenna dynamics, defining a priori model is difficult to get. Given input and output data, artificial neural networks (ANNs) is capable of identifying underlying relationship between the input and output data. Some identification results are shown and compared with the results of conventional prediction error method. The results show the validation of the ANN approach for identification of the 10$\mathrm{m}$ antenna dynamics.

We use a neural network inverse model for control the large flexible antenna. In the neural network inverse model, a neural network is trained, using supervised learning, to develop an inverse model of the antenna. The network input is the process output, and the network output is the corresponding process input. The control results show the validation of the ANN approach for identification and control of the 10-m flexible antenna.

\section{Antenna Structure and Measurement System}

10-m antenna structure is shown in Figure 2. Ukita and Ikeda made experiments on a 10-m antenna of Nobeyama Radio Observatory with accelerometers and angle encoders. The angle encoders have a 25-bit resolution $\left(\mathrm{LSB}=0.039^{\prime \prime}\right.$ ) and were measured to have an accuracy of $0.03^{\prime \prime}$ rms. The drive system of the antenna under no wind disturbance has been measured to have servo errors of typically $0.04^{\prime \prime}$ and $0.10^{\prime \prime} \mathrm{rms}$ for rotational velocities $<0.001 \mathrm{deg} \mathrm{s}^{-1}$ and $0.1-$ $0.001 \mathrm{deg} \mathrm{s}^{-1}$, respectively. The telescope was located at a highland of $1350 \mathrm{~m}$ elevation. There are three piezoelectric seismic sensors (PCB Model 393B12) at a subreflector mount chassis, four beneath a panel support board of the backup

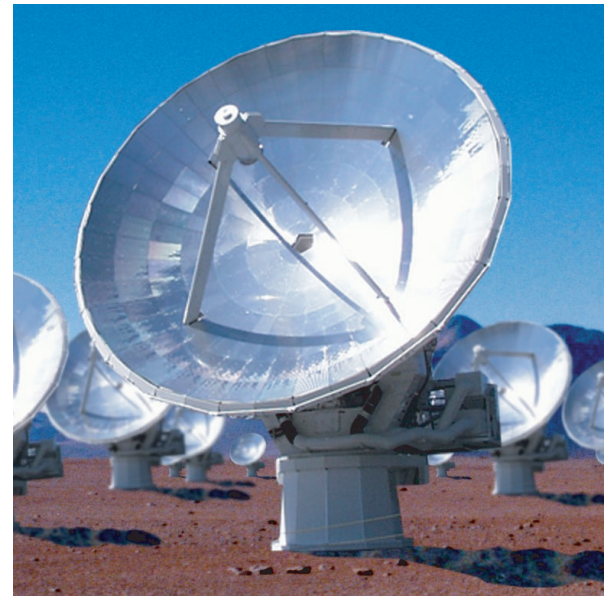

Figure 2: Antenna structure.
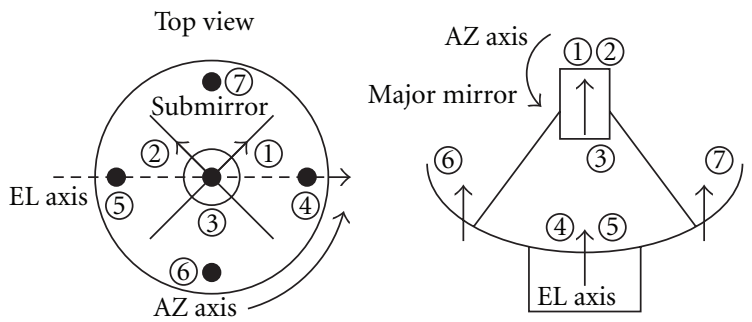

FIgURe 3: Sensors and encoders position.

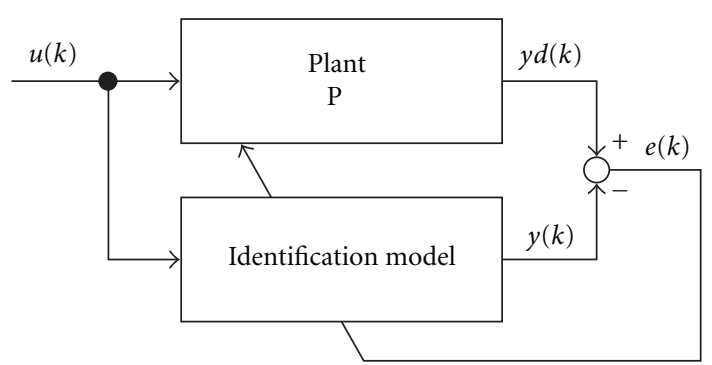

FIgURE 4: Identification system.

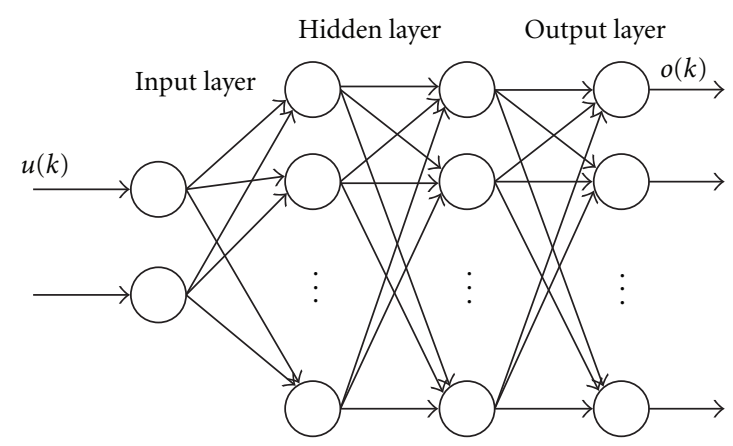

FIGURE 5: Multiple layers neural networks. 


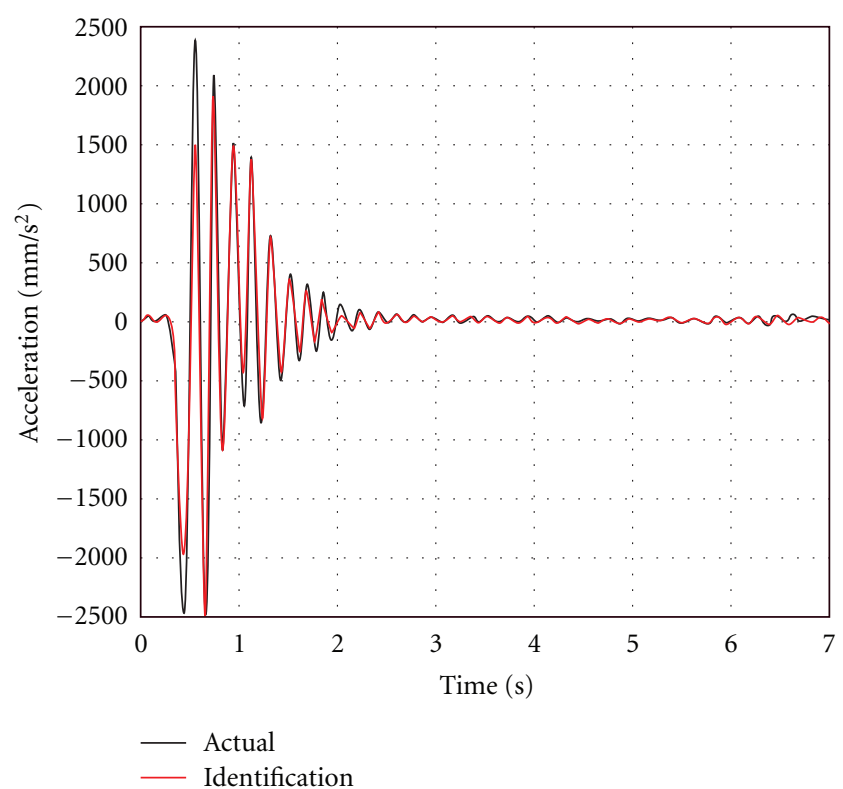

(a) Sensor 1

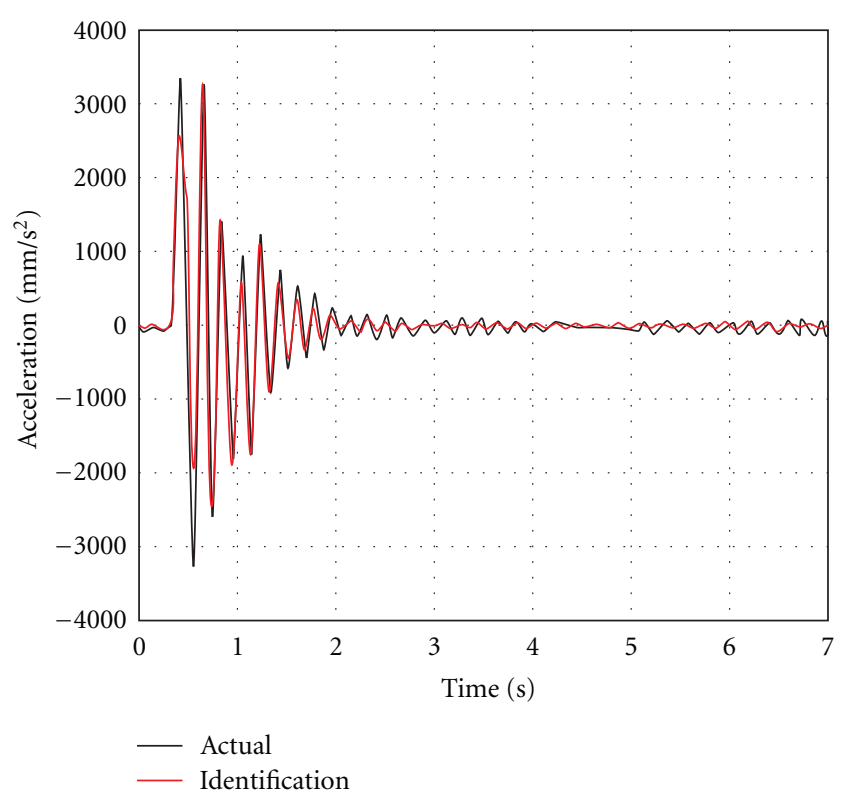

(b) Sensor 5

FIGURE 6: NN identification results.

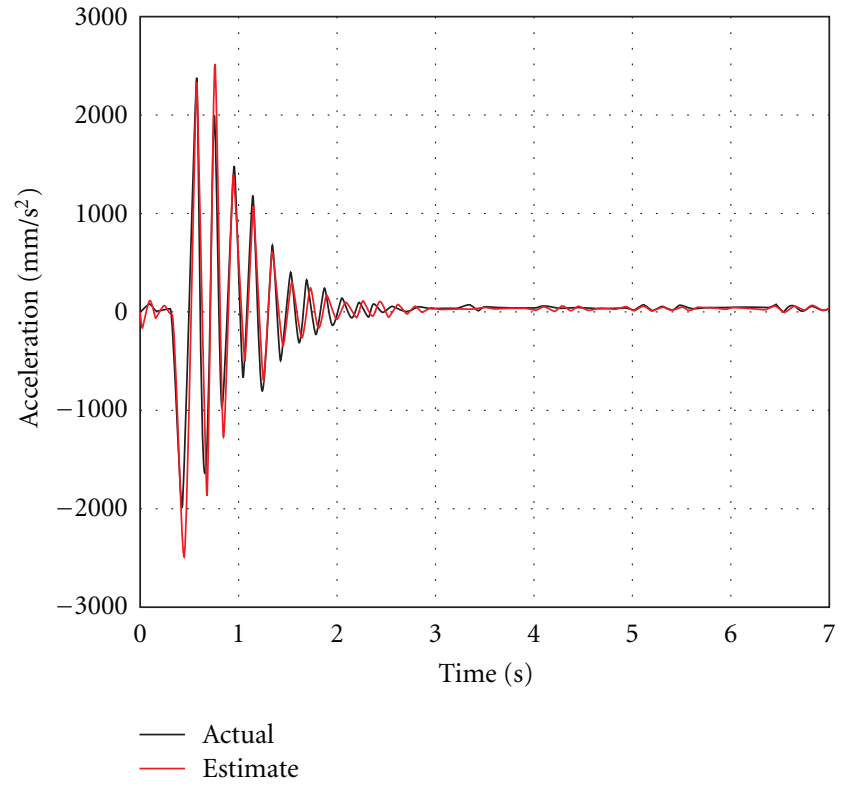

(a) Sensor 1

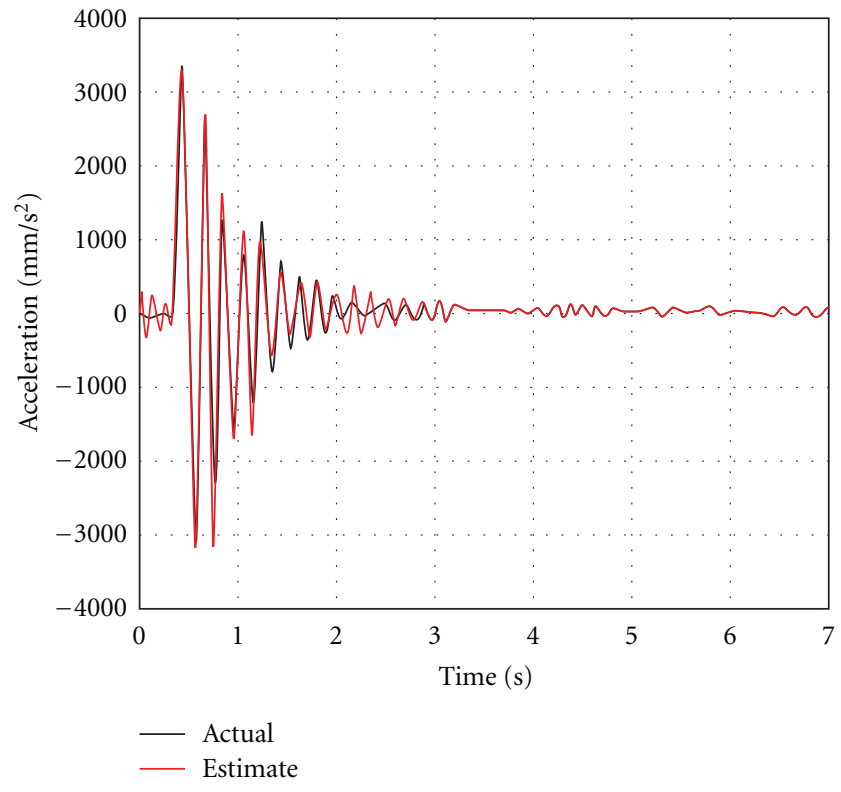

(b) Sensor 5

Figure 7: Identification using PEM.

structure (BUS), normal to the surface, one at a reference point near the center of the BUS, and four capacitive accelerometers (PCB Model 3701G3) at yoke arm ends (horizontal directions, perpendicular and parallel to the elevation axis). These data sampled simultaneously are combined to figure out the oscillations of antenna global structure. For example, differences between pairs of sensors in the BUS tell us a tilt motion of the dish in the reflector axis. The system had a noise floor of $3^{-8} \times 10^{-4}\left[\mathrm{~m} \mathrm{~s}^{-2} /\right.$ root $\left.\mathrm{Hz}\right]$ in the 0.1 to $1 \mathrm{~Hz}$ band and $2 \times 10^{-4}\left[\mathrm{~m} \mathrm{~s}^{-2} /\right.$ root $\left.\mathrm{Hz}\right]$ in the frequency range from 1 to $20 \mathrm{~Hz}$ under a condition of no wind. Our 16-bit ADC has 16 single-ended input channels with a bipolar input range of \pm 5 Volt, and makes negligible contribution to the noise floor. Comparisons between Fourier spectra of the sensor outputs under windy and no-wind conditions suggest that the components below $0.7 \mathrm{~Hz}$ seem to be due to noise (poor stability) of the sensors and/or measurement system. Sensor outputs and angle 


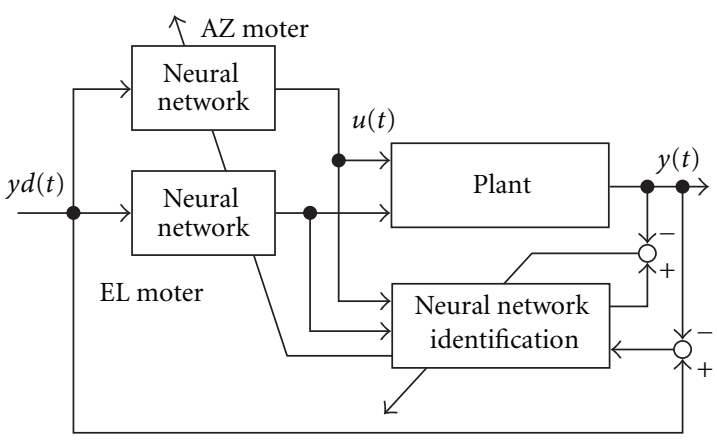

FIgURE 8: Control system.

encoder readouts were simultaneously recorded at a rate of $100 \mathrm{~Hz}$, while the antenna was driven at a rotational speed of $10^{-5} \operatorname{deg~s}^{-1}$ and was pointed at various wind attack angles under a windy condition of typically $10 \mathrm{~m} \mathrm{~s}^{-2}$ (Figure 3 ).

\section{Identification Using Prediction Error Method}

Control requirements can narrow the regions of time frequency over which an adequate model fit is necessary.

Therefore, if the control requirements are incorporated in the parameter estimation problem, it becomes possible to obtain improved models over the frequency band which is of importance to the control problem.

This is the objective of the control-relevant parameter estimation problem.

In a more generalized mathematical sense, the controlrelevant parameter estimation problem is interpreted an optimization problem which requires minimizing a functional of the weighted error between the true and estimated plant models.

Parametric identification methods are techniques used to estimate parameters in given model structures.

It is basically a matter of finding those numerical values of parameters that give the best fit between the model output and the measured one.

System identification is concerned with the building of dynamic models which describe the relationships between measured signals. The system identification problem is to estimate the model of a system based on observed inputoutput data. Here, the parametric identification methods are used. Parametric identification methods are techniques used to estimate parameters in given model structures. It is basically a matter of finding those numerical values of parameters that give the best fit between the model output and measured one. The applied parametric model is the ARMAX (auto-regressive moving average eXogeneous) model which corresponds to the description [10]

$$
A(q) y(t)=B(q) u(t-n k)+C(q) e(t)
$$

where $q^{-1}$ is the delay operator, $n_{k}$ is the time delay, and $A(q), B(q)$ is

$$
\begin{aligned}
& A(q)=1+a_{1} q^{-1}+\cdots+a_{n_{a}} q^{-n_{a}}, \\
& B(q)=b_{1} q^{-1}+\cdots+b_{n_{b}} q^{-n_{b}} \\
& C(q)=1+c_{1} q^{-1}+\cdots+c_{n_{c}} q^{-n_{c}} .
\end{aligned}
$$

A time domain description of the system is given

$$
y(t)=G(q) u(t)+H(q) e(t) .
$$

Given a description (1) and having observed the inputoutput data $u, y$, the prediction errors $e(t)$ in (1) can be computed as:

$$
e(t)=H^{-1}(q) y(t)-H^{-1}(q) G(q) u(t) .
$$

These errors are, for given data $y$ and $u$, functions of $G$ and $H$. The prediction error method is used in order to determine estimates of $G$ and $H$ by minimizing

$$
V(G, H)=\sum_{t=1}^{N} e(t)^{2} .
$$

\section{NN Identification}

The most popular control system application of neural networks is also the most straightforward conceptually. The supervised learning capabilities of neural networks can be used for identifying process models from input/output data. The process data are the training set for the network, the weights of which are adjusted until the network model output accurately predicts the actual process output. Once the training process is successfully concluded, the neural network constitutes a black-box, nonparametric process model [11].

Figure 4 is an identification system used in this paper. This system produces output $y d(k)$ which approximates $y(k)$ when subjected to the same input $u(k)$ as the plant. $u(k)$ are AZ axis and EL axis angle data. $y d(k)$ are accelerometer's output data on the submirror and the major mirror of the antenna. $y(k)$ is the prediction value of the output in the identification model. $e(k)$ is an error which $y d(k)$ is compared with $y(k)$. Identification model structure is multiple layers artificial neural networks (ANNs). The ANNs have an input layers, an output layer, and two hidden layers as shown in Figure 5. This ANNs have two inputs and seven outputs. The ANNs is a feed forward type including no combination inside the layers. Each hidden layers have thirty units. Each neuron activation function $f\left(\right.$ net $\left._{i}\right)$ is the sigmoid function given as

$$
\text { out }_{i}=f\left(\text { net }_{i}\right)=\frac{1}{1+\exp \left(- \text { net }_{i}\right)} .
$$

The neuron activation function of output layer is assumed to be the linear function

$$
f_{0}\left(\text { net }_{i}\right)=\text { net }_{i}
$$




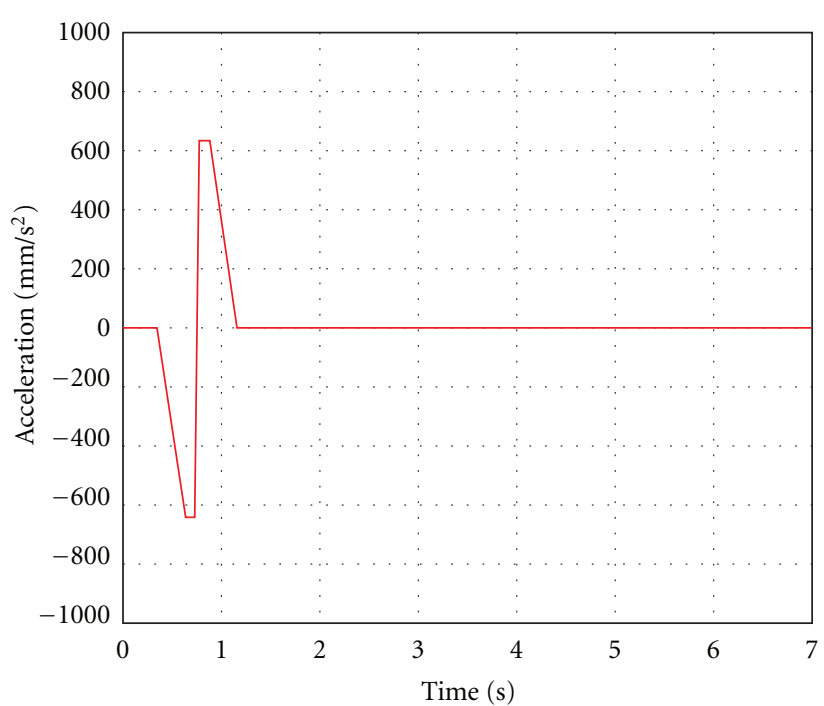

(a) Desired acceleration of sensor 1

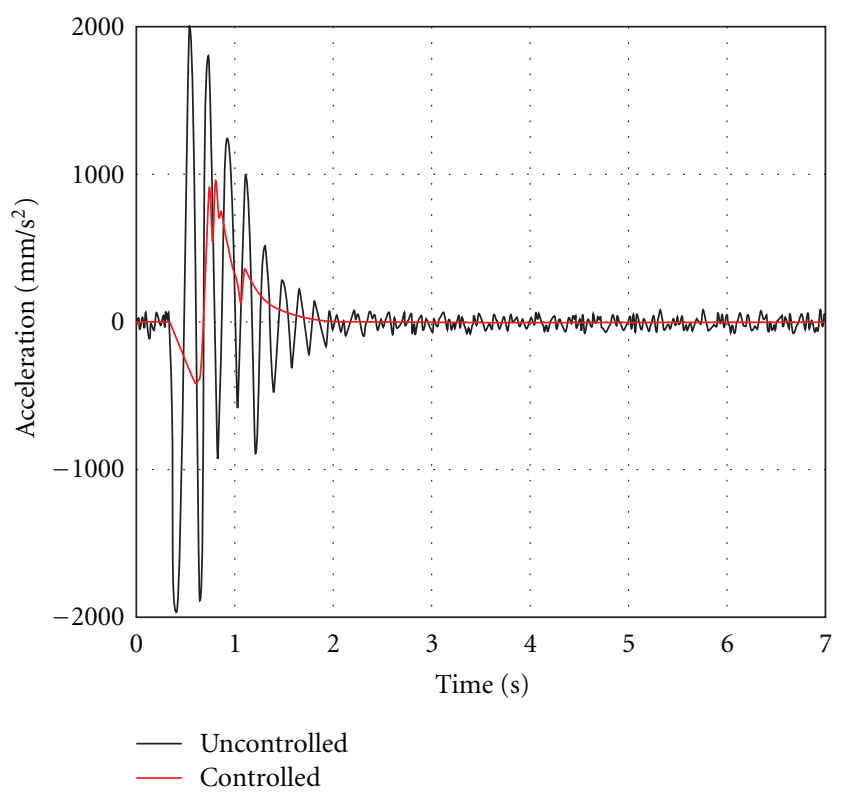

(c) Sensor 1

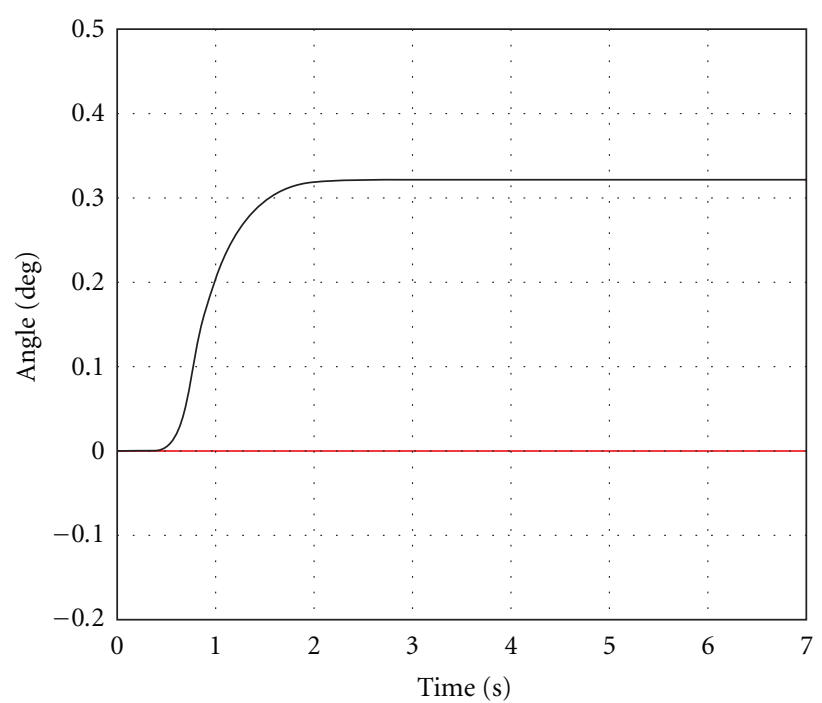

- AZ moter

(b) Input trajectory

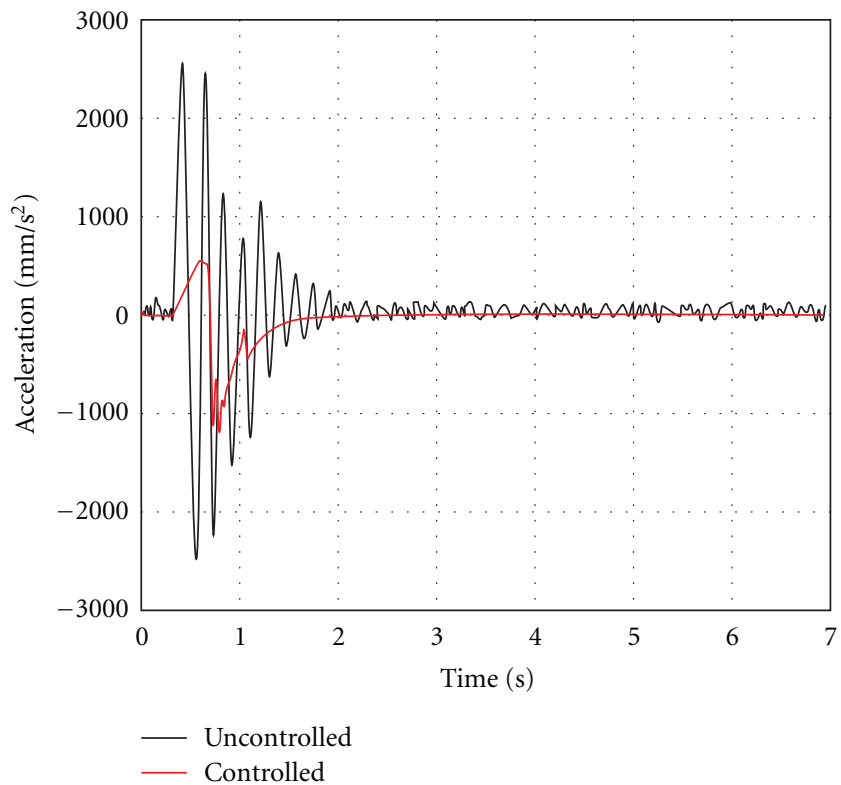

(d) Sensor5

FIgURE 9: Simulation result using error learning.

To minimize the cost function

$$
E(k)=\frac{1}{2}(y d(k)-y(k))^{2},
$$

the updating equation of the weights is defined as

$$
w_{i j}(k+1)=w_{i j}(k)-\eta \frac{\partial E(k)}{\partial w_{i j}(k)},
$$

where $w_{i j}$ is the weight value located between nodes $i$ and $j, t$ is the present iteration, and $\eta$ is the learning rate parameter. The learning rate parameter is 0.08 . The thresholds is 0.01 .

\section{Identification Results}

Identification results are shown in Figures 6 and 7. Sensor outputs and angle encoder readouts is simultaneously recorded at a rate of $100 \mathrm{~Hz}$, while the antenna is driven that the EL axis is fixed at $10 \mathrm{deg}$ and the $\mathrm{AZ}$ axis is turned around from 0 deg to 0.3 deg. 2 angle encoders data and 7 accelerometers data are used for the input data. NN identification results are shown in Figures 6(a) and 6(b). Identification results of the prediction error method are shown in Figures $7(\mathrm{a})$ and 7(b). The blue line shows the actual experimental result, and the green line shows the identification result. 


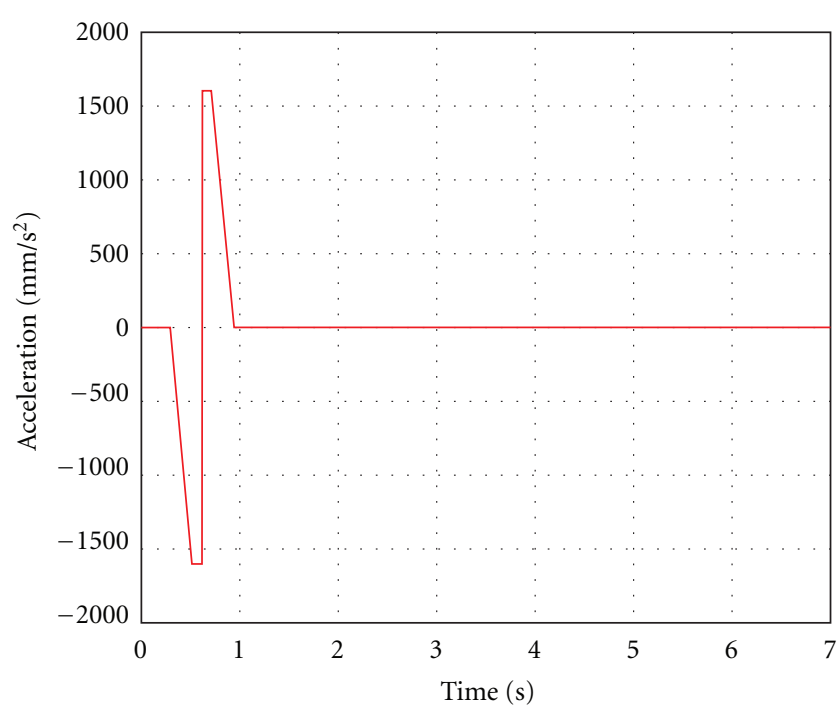

(a) Desired acceleration of sensor 1

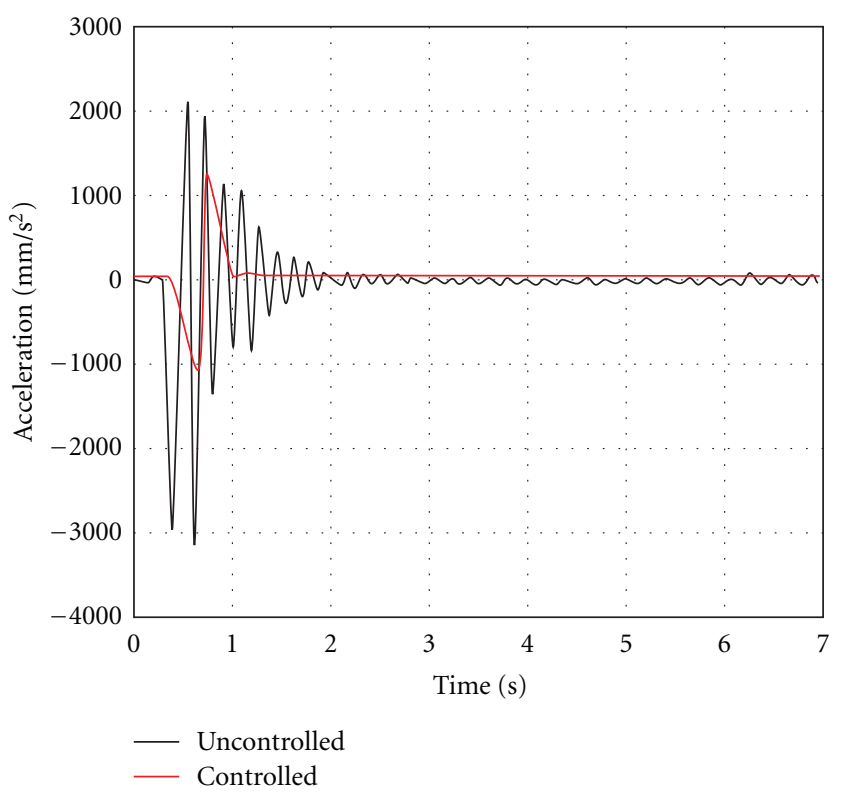

(c) Sensor 1

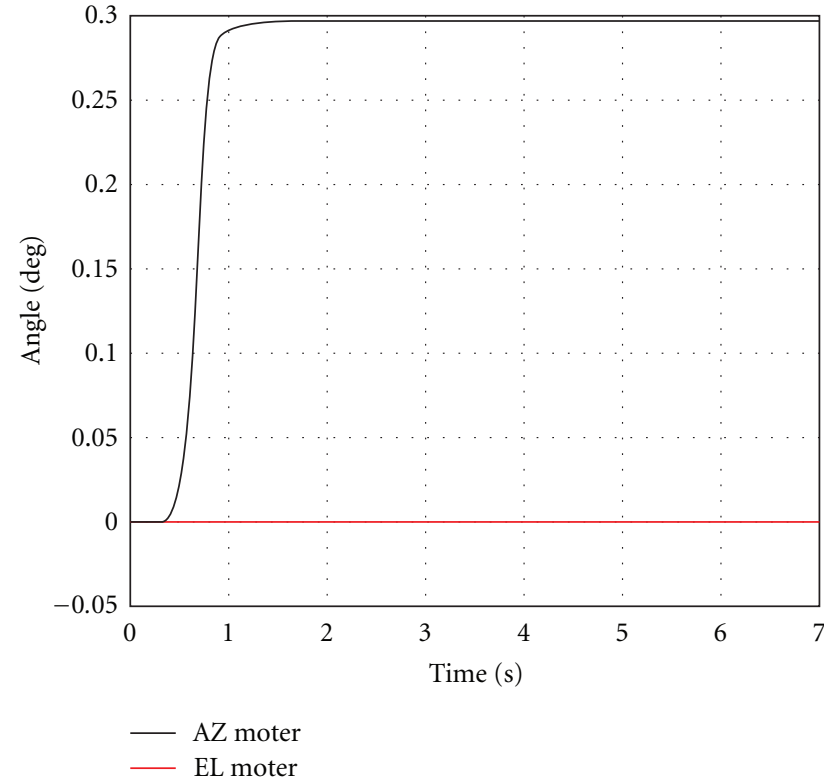

(b) Input trajectory

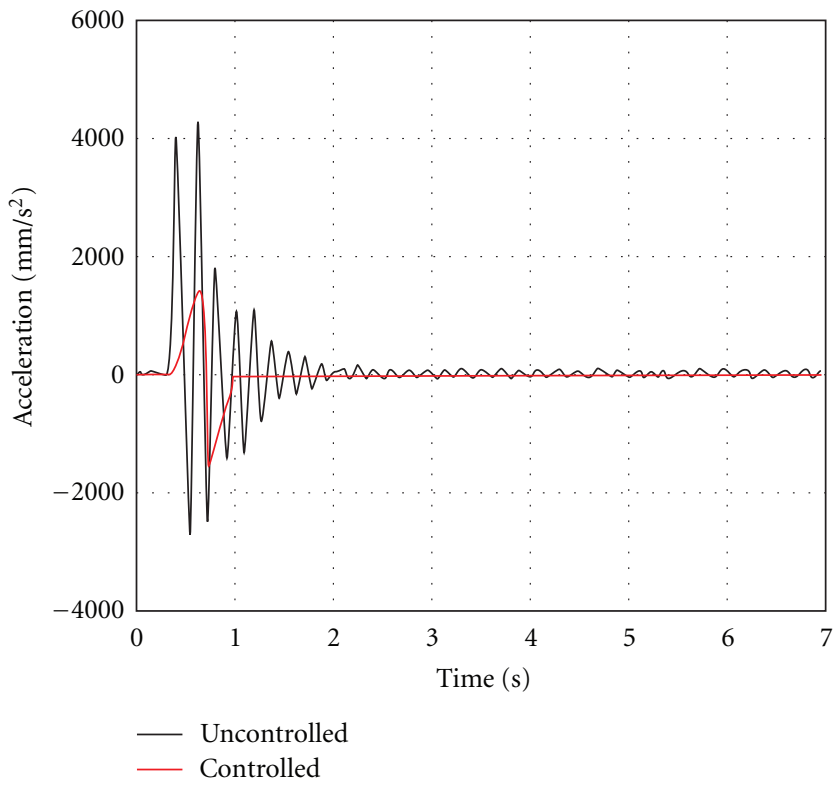

(d) Sensor5

FigUre 10: Simulation result using Jacobian learning.

Lower-frequency vibration modes have good accuracy, but higher-frequency vibration modes have not good accuracy. We carried out a computation by using MATLAB system identification toolbox. Identification in prediction error method is two inputs and 1 output. Identification model is determined in encoder input and each accelerometer sensor output. Compared with prediction error method and NN identification method, one cycle squared estimation error of the NN method is smaller than that of the prediction error method. One cycle squared estimation error of the prediction error method of the sensor no. 1 is $7.666 \times 10$, that of NN identification method of the senor no. 1 is $1.9 \times 10$, that of the prediction error method of the sensor no. 3 is $4.8794 \times$ 10 , and that of the $\mathrm{NN}$ identification of the sensor no. 3 is $3.898 \times 10$, respectively. The total squared error of the seven sensors of NN identification method is $21.1732 \times 10$.

\section{NN Controller}

The inverse model of a dynamical system yields input for given output. The model is a crucial role in a range of control structures. Conceptually, the simplest approach is direct inverse modeling. A synthetic training signal is introduced to 


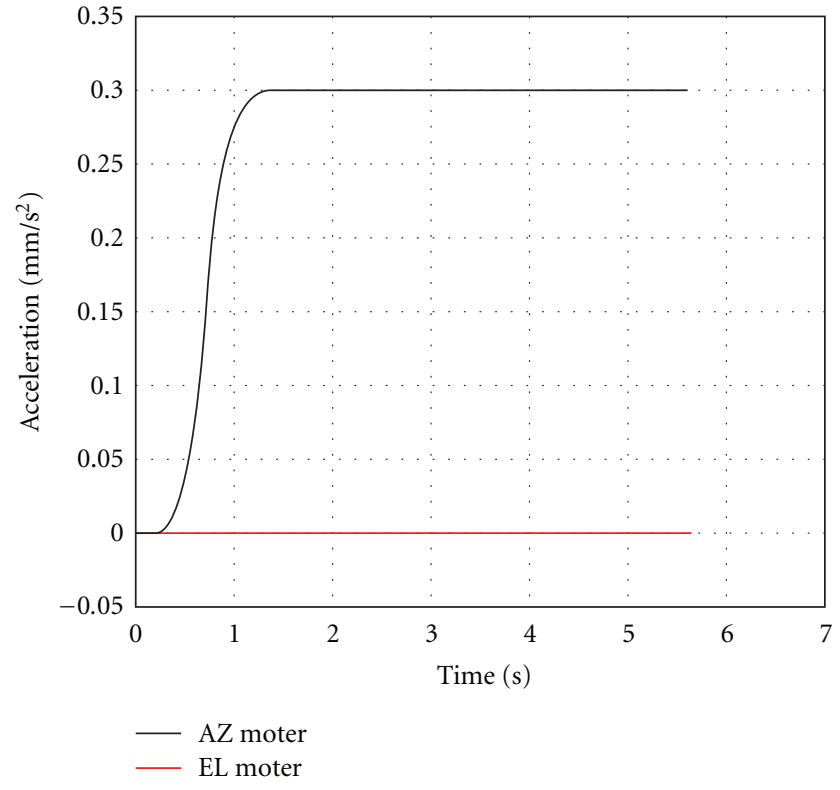

(a) Input trajectory

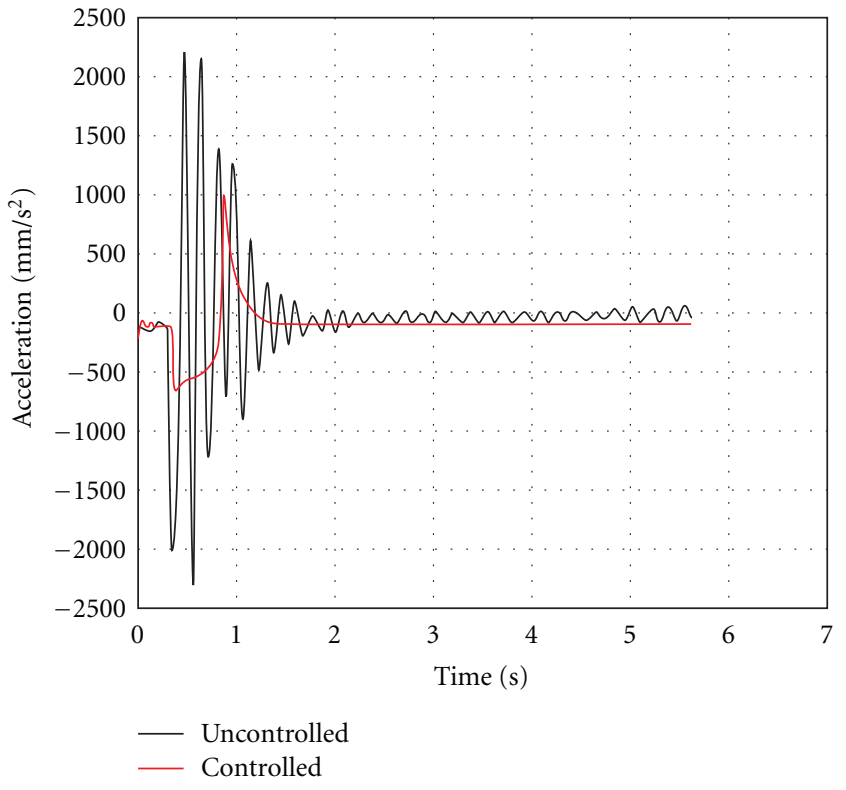

(b) Sensor 1

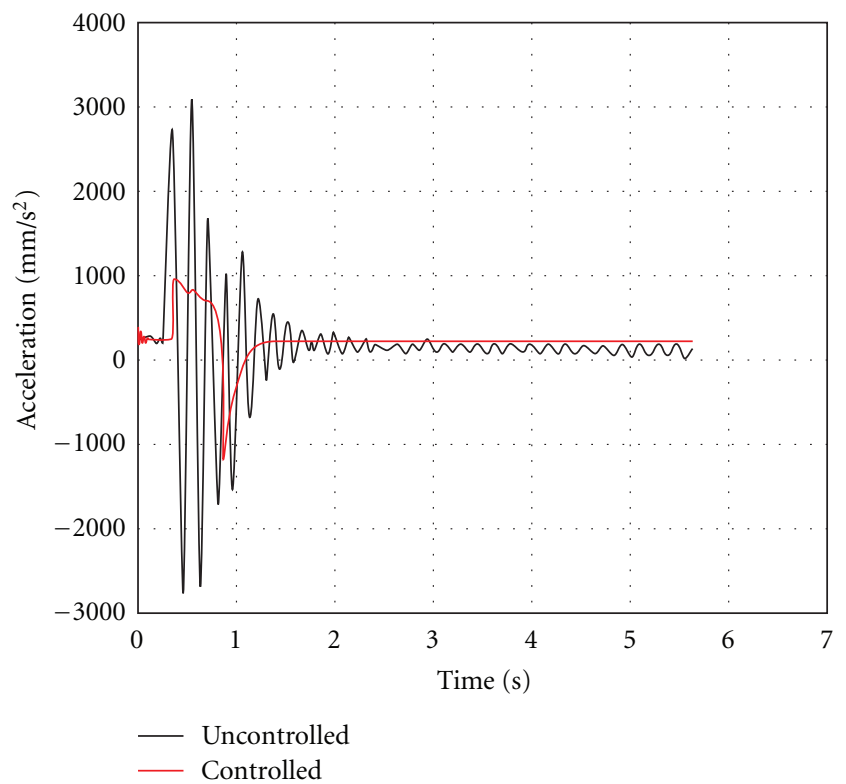

(c) Sensor2

FIGURE 11: Simulation results using vector and acceleration value.

the system. The system output is then used as input to the network. The network output is compared with the system input and this error is used to train the network. This structure will clearly tend to force the network to represent the inverse of the plant [12]. Figure 8 shows the control system using NN. In order to more precisely track the desired trajectory, we developed a new cost function to associate with the learning process of the networks

$$
E(k)=\sum_{i=1}^{7}\left\{\frac{1}{2} q\left(y o_{i}(k)-y_{i}(k)\right)^{2}+\frac{1}{2} p(u o(k)-u(k))^{2}\right\},
$$

where $q$ is a weight of acceleration output, $p$ is a weight of desired trajectory input. We can rewrite the updating equation as

$$
\begin{gathered}
\frac{\partial E(k)}{\partial w_{i j}(k)}=\frac{\partial E(k)}{\partial u(k)} \frac{\partial u(k)}{\partial w_{i j}(k)}, \\
\frac{\partial E(k)}{\partial u(k)}=-q\left(y o_{l}(k)-y_{l}(k)\right) \frac{\partial y(k)}{\partial u(k)}-p u(k),
\end{gathered}
$$

where $\partial y(k) / \partial u(k)$ is the system Jacobian.

Figures 9 and 10 show the controlled and non controlled results of the accelerometers output of the sensor no. 1 and 
no. 5. Figure 9 shows the results of the conventional error learning. Figure 10 shows the results of the Jacobian learning. Figure 10(a) shows the desired acceleration of the sensor no.1 and no.5. Figure 10(b) shows the desired trajectory of the azimuth axis and the elevation axis. The residual vibration with the NN feed-forward controller is suppressing more quickly than that without the controller. Compared with the results of he conventional cost function (8) and those of the new cost function (10), the new cost function is more effective for suppressing the residual vibration and tracking the desired trajectory (Figure 11).

\section{Conclusion}

Identification and control of a 10-m antenna dynamics for the Atacama large millimeter array project using accelerometers and angle encoders data were presented. Compared with prediction error method and NN identification method, one cycle squared estimation error of the NN method was smaller than that of the prediction error method.

A neural network inverse model was used for control of the large flexible antenna. In the neural network inverse model, a neural network was trained, using supervised learning, to develop an inverse model of the antenna. The network input was the process output, and the network output was the corresponding process input. The control results showed the validation of the ANN approach for identification and control of the 10-m flexible antenna, especially the system Jacobian learning.

\section{Acknowledgment}

This work was supported in part by the ALMA cooperative research project.

\section{References}

[1] W. K. Gawronski, C. S. Racho, and J. A. Mellstrom, "Application of the LQG and feedforward controllers to the deep space network antenna," IEEE Transactions on Control Systems Technology, vol. 3, no. 4, 1995.

[2] N. Ukita et al., "NRO 10-m submillimeter telescope," in Radio Telescope, H. R. Butcher et al., Ed., vol. 4015 of Proceedings of the SPIE, pp. 177-184, 2000.

[3] N. Ukita et al., "A high-precision angle encoder for a 10-m submillimeter antenna," National Astronomical Observatory of Japan, vol. 6, pp. 59-64, 2001.

[4] J. W. Lamb, "Optimized optical layout for MMA 12-m antennas," Memo 246, NRAO MMA Memo Series, 1999.

[5] D. P. Woody and J. W. Lamb, "A design for a precision 10m sub-millimeter antenna," Memo241, NRAO MMA Memo Series, 1999.

[6] M. A. Holdaway, "Fast switching phase correction revisited for 6412 m antennas," ALMA Memo 403, 2001.

[7] M. A. Holdaway, "How many fast switching cycles will the MMA make in its lifetime?" MMA Memo 174, 1997.

[8] W. Gawronski, "Antenna control systems: from PI to $\mathrm{H} \infty$," IEEE Antennas and Propagation Magazine, vol. 43, no. 1, 2001.

[9] L. R. D'Addario and D. T. Emerson, "On-the-fly fringe tracking," ALMA Memo 331, 2000.
[10] L. Ljung, System Identification: Theory for the User, Prentice Hall Information and System Sciences Series, Prentice Hall, 2nd edition, 1999.

[11] K. S. Narendra and K. Parthasarathy, "Identification and control of dynamics systems using neural networks," ICASE Journal, vol. 1, no. 1, pp. 123-126, 1996.

[12] M. I. Jordan, "Generic constraints on underspecified target trajectories," in Proceedings of the International Joint Conference on Neural Networks I, pp. 217-225, 1989. 

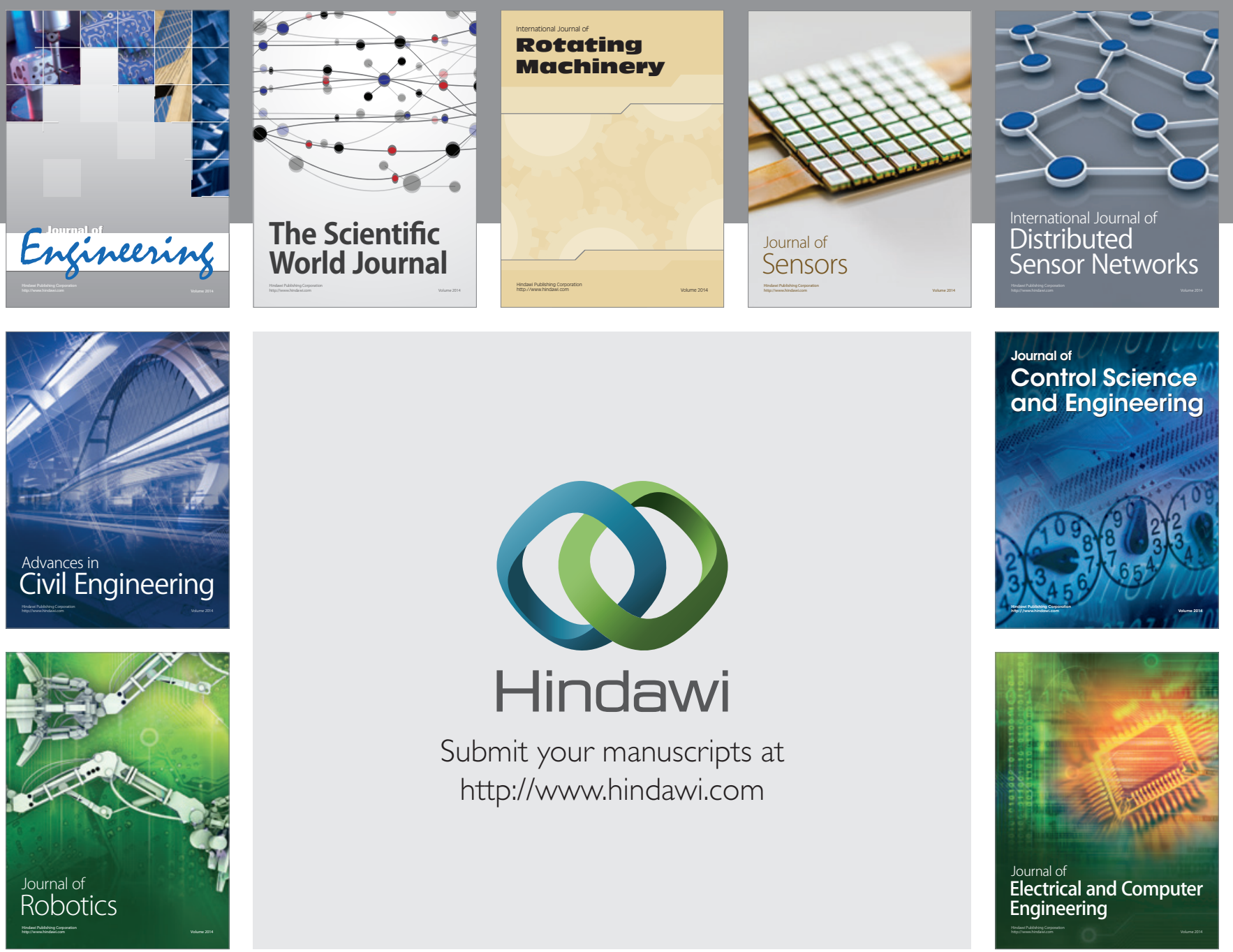

Submit your manuscripts at

http://www.hindawi.com
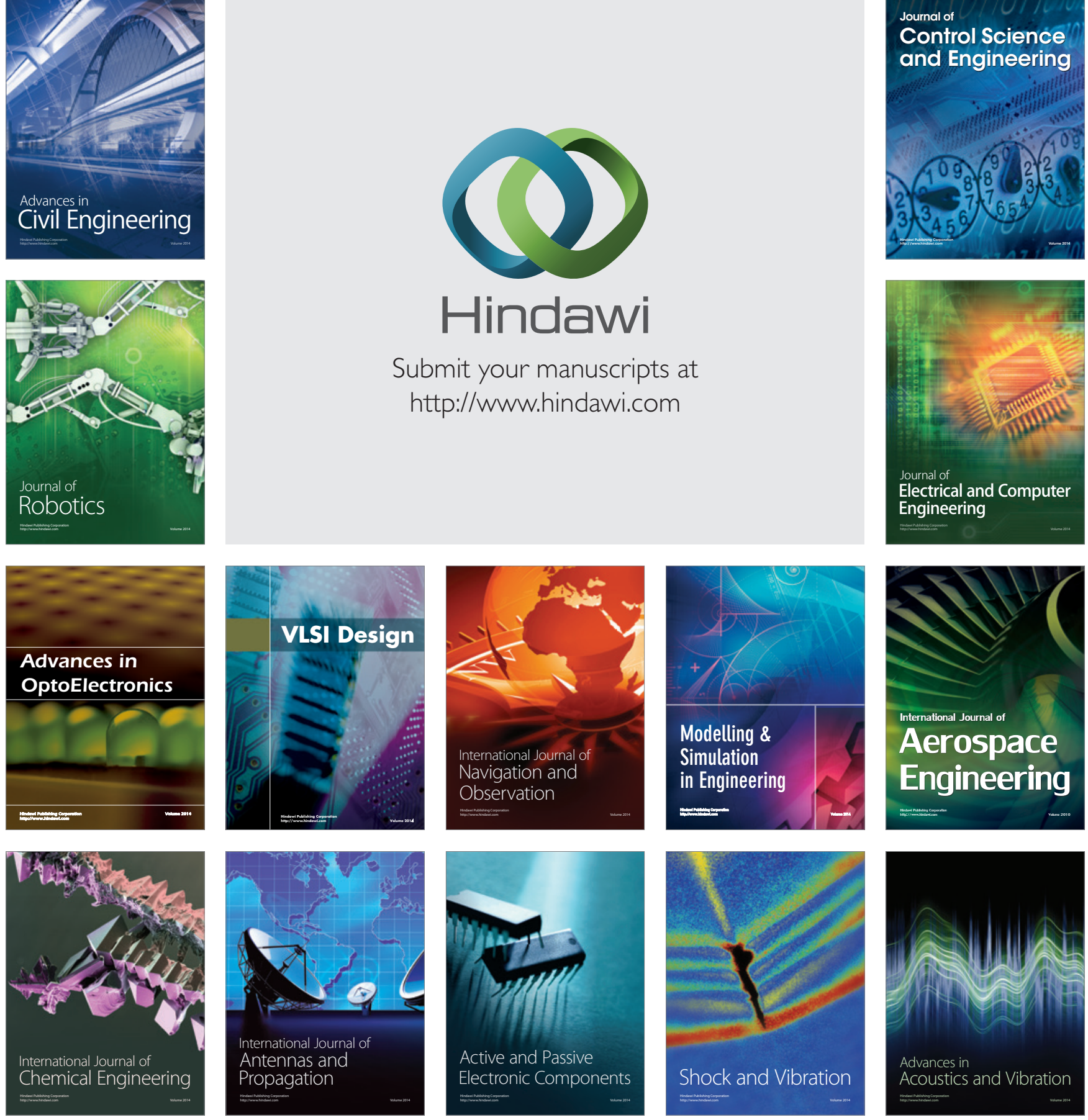